\title{
A Systematic Literature Review of New Trends in Self- expression Caused by Emojis and Memes
}

\author{
Victor Huang ${ }^{1, \dagger}$, Yifan $\mathrm{Hu}^{2, \dagger}$, Yaohua $\mathrm{Li}^{3, *, \dagger}$ \\ ${ }^{1}$ International department of Chengdu foreign language school \\ ${ }^{2}$ The High School Affiliated to Beijing Normal University \\ ${ }^{3}$ China University of Mining and Technology \\ ${ }^{*}$ Corresponding author. Email: 11184034@cumt.edu.cn \\ These authors contributed equally.
}

\begin{abstract}
Emoji and memes are a new way to present one's emotion, are popular among young people. It is vital nowadays because almost everyone today largely relies on social networks and sends emojis and memes. Lots of research about emoji and memes have been conducted. Here, this paper summarizes new trends brought by their appearance in China. The article is written using the method of literature review. Based on the research, this paper reveals three typical trends of selfexpression in China society: emoji and memes create new ways to shape one's self-image because of their characteristics and the "looking glass self" effect; emojis and memes cause generation gaps due to physical difference and different growth background; emoji and memes lead to obscure boundaries between diverse social circles. The research about emojis and memes enables us to know more about why people can easily use emojis and memes to shape a persona and why there are differences between elderly groups and young groups when they use emojis and memes, and why the use of emoji blurs the social sphere. By doing that, this paper, from a micro perspective, examines the trend of each case and examples as individual trends. Also, from a macro perspective, this paper shows that emojis bring infinite possibilities to society, enrich people's social channels, and ultimately affect the structural changes of social circles.
\end{abstract}

Keywords: China, Emojis and memes, Self-expression

\section{INTRODUCTION}

Expressions are a social tool that permeates every minute of our social lives. In today's society, at least one out of every ten messages contains an emoticon. It helps people express themselves better [1]. The essential traits of expressions are perceptual intuition, effectiveness, interest [2]. They showed that expressions could improve the efficiency of users' communication and interaction and enrich users' communication forms, thus making it a famous network landscape on social platforms. Compared to the past period when emojis and memes did not appear and when people depended pretty much on face-to-face communication, today, memes and emojis are prevailing in our country [3].

This phenomenon leads to three new trends in our society. The first trend is that with the popularity of online communication and images to convey one's emotion, it is easier now to create one's persona. In other words, people are easier today to pretend what their personalities are. Moreover, In the use of emojis and memes, people in different generations have formed different culture styles between age levels due to differences in life experience, lifestyle, cultural taste, and different cultural communication contents and characteristics. The so-called "generation gap" is directly reflected in distinct differences of image form, expression meaning, and language style of emojis and memes used by middle-aged and elderly people and young Internet users [4]. In addition, facial expressions and body postures play a significant role in communication in people's daily lives. When the communication between the two chatters is not the same, people can use facial expressions or body language to express the information they want to convey. Emojis and memes have the same benefit in a similar way and form that express emotions more vividly and obscure the social circle's boundaries.

No trends can be discussed without cultural context. In both China and Western countries, emojis and memes 
serve as an important way to directly show one's emotion and attitude toward the person he is talking to. Expressions are one of the essential parts of social life in both China and western society today. The first difference is that in the Western world, they separate the expressions into two parts—memes and emojis, they have different usages. However, the Chinese collectively call them expressions, and this research focuses on the new trends of this young culture, not one specific expression. On the other hand, although there is also a generation gap in emojis and memes in Western society, emojis and memes mainly exist in young people. Compared with China, emojis and memes do not seem to be popularized to the middle-aged and elderly in foreign countries.

This study aims to sort out three social change trends brought about by emojis to reflect the significant impact of emojis on the social communication field in China. These three new social trends make our social lives more fulfilling and exciting. We want to know precisely how they happen and what they influence our lives. To achieve this goal, the main body of this paper is divided into three parts: increasing new image-building ways, aggravating the generation gap, and blurring the social circle. These three parts sort out the young social trend changes brought by expressions from three dimensions.

\section{METHODS AND MATERIALS}

The methods used in the paper are mostly literary reviews. The materials are searched online in some databases. They include factual information, analysis, and general trends. Based on this information, this paper future concludes what three new trends of selfexpressions bring and why the trends are brought.

\section{THREE NEW TRENDS OF SELF- EXPRESSION CAUSED BY EMOJIS AND MEMES}

\subsection{Memes and emojis help people to create their persona online}

Emojis and memes are thought to shape one's personality or pretend one's character. To understand why emojis and memes have such function, we have to know their commonly-held features. Most emojis and memes are simple, intuitive, humorous, personalized, and vivid [5]. In other words, everyone can quickly and directly understand what the emojis and memes want to convey, and emojis and memes can express emotions that can't be conveyed by words that are not vivid. However, despite the superficial meanings, most emojis and memes have hidden meanings, incredibly essential to building one's personality. The appearance of emojis avoids the monotony of pure text communication to some extent, makes up for the deficiency of visual experience, and provides important supplementary information in attitude, emotion, voice, and other aspects. Emoticons can arouse people's association and reflect the connotation and extension of things [6]. With icons, people who receive emojis and memes can have solid and vivid impressions in their minds.

Another reason why emojis and memes help us build our personality is reflected in the concept of looking grass the self. According to the theory proposed by American sociologist Cooley, individual behavior largely depends on the understanding of "self," which is mainly formed through social interaction with others, and others' attitude or evaluation of oneself, which is a mirror of "self" [7]. The use of memes of one individual is the attempt to show his understanding of "self," and the feedback given by the one who receives the emojis and memes is served as the "mirror." The interaction of "mirror" and "self" further helps people know who they are and what roles they should play in modern society. Therefore, emojis and memes can not only shape a fake impression or persona but can help people understand what they should do, who they are, and what persona they want to have. Emojis and memes help people know what they want, which allows them to build a persona.

According to one research, to understand users' use of emojis, one author issued a questionnaire survey. The questionnaire was open for ten days, and a total of 460 valid questionnaires were collected. The questionnaire results show that $30.6 \%$ of people choose to use emoticons for self-image building, and $16.9 \%$ of them choose emoticons as their social profile pictures, which indicates that emoticons are not only an expression tool used in daily communication but also a meaningful way to build personal image and maintain social relations [8]. This research strongly supports the idea that emojis and memes are beneficial. They help people shape their personality by asking the opinions of the public or the user who use emojis and memes. The result also shows that the character shaped by emojis and memes is not intentional but may be purposeful.

Another example is called the "Diba expedition." "Diba" refers to Li Yiba in Baidu Tieba, one of the most popular Tieba, with more than 20 million members and 800 million posts. The leading members of this "Diba expedition" also come from this source. Therefore, it is undoubtedly a group communication behavior with a clear goal and relatively rational, and the communication carrier is emoji. In this virtual community, the identity of "self-categorization" solidifies the similarities between the members of the inner group and the differences between the members of the outer group. The selfdeprecating phenomenon of "Diaosi" that has attracted the attention of the linguistics and social circles is derived from "Diaosi" [9]. In this situation, everyone in Diba uses similar memes, and this similarity leads to selfcategorization, which corresponds to the "mirror self" 
theory. Because people in Diba use memes and receive positive feedback from other people, they classify themselves as members of Diba and then follow the rules of Diba. Eventually, everyone in Diba gains a similar personality and one organization where everybody has identical persona forms.

\subsection{The generation gap in the usage of emoji and memes}

With the popularity of the Internet and smartphones, many middle-aged and older adults are contacting the Internet, and social media such as WeChat is their first stop. The middle-aged and elderly memes with middleaged and elderly groups as the primary users are generated. $\mathrm{Hu}$ believes that middle-aged and elderly memes have some remarkable characteristics. First, these memes have a high saturation, high contrast, and gorgeous colors. Red, yellow, blue, and green are the primary colors of memes for middle-aged and older adults, while cold colors such as black, white, and gray are rare. Secondly, the characters on these memes are significant, and the font size is changeable. Different from the multiple connotations of the content of the text on the young people's emojis and memes, the content of the middle-aged and elderly memes is regular and literal.

What's more, the patterns on these memes are also very particular. First, flowers and children are regarded as beautiful symbols by middle-aged and older people; Second, it may be because the middle-aged and older people are unwilling to belittle themselves as animals. Compared with the expression bags of cocky dogs and kittens, which are popular among young people, middleaged and older adults prefer blooming peonies, charming roses, and lovely children. Finally, the emotional expression of the memes of the middle-aged and elderly is very positive. For instance, famous aphorisms, encouraging words during the new year and festivals, and so on. It can be seen that the memes of middle-aged and older adults are much different from memes used by young people [9].

So why is there such a big difference? Liu and Qian have put forward some professional views on this, which I summarize into two parts. The first is the physiological difference. As people get older, people's sensory organs will gradually decline. On the one hand, the adaptability to light and shade decreases. On the other hand, the perception and discrimination of color decrease with age. The deterioration of physiological function enhances the needs of middle-aged and older adults for bright colors and solid visual perception [10]. The second is the difference in growth background. In the 1980s, graphic design posters usually had text interpretation content, which was a concrete and vivid description of reality or the past, including great rivers and mountains of China, positive life of people, and other content. These expressions have similarities in content and visual form with today's middle-aged and elderly memes. With this background, their inherent visual culture is quite different from the visual culture accepted by contemporary young people. Even if the graphic design in modern life is more abundant, the absorption ability of middle-aged and elderly groups to new aesthetics has been reduced, and what remains in the subconscious is still the inherent aesthetics so that such differences will occur [11].

What impact will such differences have on family intergenerational relations? Liu and Li have carried out researches on this. Firstly, with the development of network society, emojis and memes have become an indispensable part of children's social chat. For example, when parents and children mention topics related to learning and work, the atmosphere is easy to become tense. If some memes are used to adjust the atmosphere in the communication, the situation will be eased, and the transmission can continue. Using memes to communicate is also a new way slowly explored by parents and children, creating a relaxed and harmonious communication context [12]. In addition, in the face of a rapidly changing society, parents often can't keep up with the pace of the development of the times. Parents want to understand their children's lives through their dialogue, but they don't know what they are talking about. This will affect the parents' sense of closeness and belonging to this society, which is not healthy to both body and mind. The memes can become the starting point for parents to reacquaint this society and open the door to communicating with the younger generation. This way can change middle-aged and older adults' mentality, regain self-confidence, and make the relationship between the two ages closer. The two generations grow together [13].

\subsection{The obscure boundaries between diverse social circles brought by expressions}

The concept of the social circle originated in modern times, but it has been a traditional phenomenon throughout whole Chinese history. In his research, Mr. Xiaotong Fei once proposed that "China's social structure is like a circle of ripples pushed out when a stone is dropped on the water. Each person is the center of the circle, pushed out by their social influence. What is pushed by the ripple of the circle is connected. Everyone may not use the same circles at a particular time and place "[14]. Since ancient times, it has been a tradition in China to take individuals as the center to reflect the degree of closeness and estrangement of social relations. Until today, with the popularization of information networks and more social media, vagueness has emerged. In addition, the emotions we studied are the most important driving force, and the blurring of the network social circle is the trend and expression of the continuous development of social life. Emotions are a tool that serves as a bridge for communication between traditional social 
circles in cyberspace, successfully transforming online communication from traditional to modern networks. According to Qianru Wang and Rong Zhang, the meaning of network circle layer mainly includes two aspects: the horizontal "circle" formed based on factors such as interest and relationship, namely various network social circle groups; The longitudinal "layers" gradually derived from inside the network circle group [15]. In the changing trend of network circles brought by today's expressions, there are mainly two types of hierarchical relationships: one is the hierarchical relationship formed by the differentiation of power levels of network resources, similar to the "superior and subordinate" relationship; The other is the individual-centered hierarchical relationship formed according to the relationship between "near and far," similar to that in traditional society. However, the emergence of emojis makes Internet users in different network circles break the focus on blood relationships, geography, or business relationship in interpersonal communication in actual society and tend to the commonality of spiritual pursuit and individual interest preference [16]. More specifically, McLuhan's concern about the physical absence of human communication was that words are written in pinyin sacrificed meaning and perception. Human contact once again craves physical presence. The body is not merely a negative determinate but a meeting of the external and internal worlds, a knot of living meaning. Merlo Ponti said that the body is the foothold from which we view the world, and perception is closely related to the body. Thus, compensatory media came into being. Movie language is "the language of facial expression and body posture." Human beings send expressions that cannot be seen by the other party in the form of expressions so that the other party can see our face to accurately and quickly perceive our emotions. Emoticons communicate with feelings and emotions through embodied nature. When you're manic, choose a snarling emoji or a tearing-down emoji. Even the middle-aged and elderly emoji "Raise a glass to everlasting friendship" can take on a physical pose. All these factors lead to the blurring boundaries of the social circles.

\section{CONCLUSION}

Through the systematic arrangement, we have presented three new social changes brought by emojis and memes, which are regarded as social tools that permeate every minute of our social lives. The first one is the trend that emojis and memes can help shape human sets. We found that because of the "looking glass self" theory and unique characters of emojis and memeshelping convey emotion vividly and conveying hidden meanings - people can use them to create their personas or fake personality. The invention of emojis and memes enables people to try different ways to express themselves and shape their self-image even without actual items. They could fully express themselves online and express themselves exceedingly, leading to fake impressions and a fake personality. Some people who have a strong desire to show themselves also may intentionally shape a wrong character. The second one is about the generation gap in the usage of emoji and memes. We found that the physical difference and different growth backgrounds caused the older adults and young generation to misunderstand the cognition of emojis and memes. This difference can even promote intergenerational relations.

Last but not least, we noticed the obscure boundaries between diverse social circles brought by expressions. The successful development of emotions has provided the world with a perfect tool to cross social circles. The massive popularity of emotions has also led to the gradual blurring of the original Chinese traditional circle communication mode, which gave the society more powerful vitality. The blurring of social circles is a new social trend in the new era of the Internet brought by emotions. This phenomenon indicates that all social media users can expand their field of making friends, which can help people better communicate with likeminded people and reduce communication costs. All these three trends have something to do with selfexpression. As an essential part of social media, memes, and emojis significantly improve people's desire to present themselves and provide a more diverse way for all kinds of people to express themselves. Memes and emojis are the progress of self-impression in the age of the Internet. Through combing the three new trends of self-expression caused by emojis and memes in the age of the Internet, we can find the significant role that emojis and memes play in the progress of self-expression in China. Also, the new status of self-expression can be reflected from the three trends about memes and emojis above. The new position of China's online selfexpression that this paper combs can provide some reference for scholars and practitioners in related fields to explore the development process of self-expression. People's desire to show themselves is becoming stronger and stronger. Only by sensitively discovering and summarizing people's behaviors and concepts about selfexpression can we enhance our understanding and take control of the Internet era through the new changes in people's ways of presenting themselves. The importance of this paper lies in systematically combing the recent trends of self-expression in China caused by emojis and memes, which has played a foundation role in the next step of in-depth researchAll these three trends have something to do with self-expression. As an essential part of social media, memes and emojis significantly improve people's desire to present themselves and provide a more diverse way for all kinds of people to express themselves. Memes and emojis are the progress of self-impression in the age of the Internet. Through combing the three new trends of self-expression caused by emojis and memes in the age of the Internet, we can find the significant role 
that emojis and memes play in the progress of selfexpression in China. Also, the new status of selfexpression can be reflected from the three trends about memes and emojis above. The new position of China's online self-expression that this paper combs can provide some reference for scholars and practitioners in related fields to explore the development process of selfexpression. People's desire to show themselves is becoming stronger and stronger. Only by sensitively discovering and summarizing people's behaviors and concepts about self-expression can we enhance our understanding and take control of the Internet era through the new changes in people's ways of presenting themselves. The importance of this paper lies in systematically combing the recent trends of selfexpression in China caused by emojis and memes, which has played a foundation role in the next step of in-depth research

\section{REFERENCES}

[1] Zhu Chuanxin. (2021). Expression pack makes communication closer. Extracurricular Chinese (23), $30-31+49$.

[2] Zheng Anyuan. (2021). From "expression" package to "emotion" package: discourse representation analysis of youth subculture groups from the perspective of network carnival. Chinese character culture (15), 171-172.

[3] Wang Yan. (2021). The value and reflection of expression pack communication from the perspective of youth subculture. Western radio and television (15), 96-97+ 109 .

[4] Hu Miamiao. (2017). The rise of middle-aged and elderly emoticons and the analysis of "generation gap" behind them -- from generational differences to cultural differences. News research guide, 8(15), $85-86+131$

[5] Zhang Jiangfu \& Fang Xiang. (2009). The characteristics of "Internet language" and its sociological thinking. Shidai Wenxue (the second half of the month) (09), 118-119.

[6] Wu Jing. (2016). Communication analysis of the development of emoji in the era of new media. Shidaiguangjia (15), 83-85.

[7] Charles Horton Cooley (1902). Human Nature and Social Order. China Publishing House.

[8] Li Ziruo. (2020). The characteristics and communication functions of network emoticons in the new media era. Today's Massmedia (02), 16-19.

[9] Yu Xiaodong \& Huang Yayin. (2016). Look at the function of emoticons in network communication from "Emperor Bar". Media Observer (05), 16-18.
[10] Qian Wei, Mou Kaiyue, Sui Jiaao, Yin Yue, Li Lan. (2021). Study on the differences in the use of wechat emojis between college students and their parents. Journal of Weifang Vocational College of Engineering, 34(01),67-70+92.

[11] Liu Yun. (2010). Aesthetic Identity and Generational Estrangement of middle-aged and old expressions. Fine Arts Literature, (01),105-106.

[12] Liu Ruiyu. (2018). Research on the influence of wechat emoticons on family intergenerational communication. Unpublished doctotial dissertation. Nanjing Normal University, Nanjing.

[13] Li Peijin. (2020). A preliminary study on the intergenerational difference of Internet emoji language. China Newspaper Industry,(10),66-67.

[14] Fang Nan.(2019). Stratification of college students' network social circle in network differential Pattern. Journal of Ningbo University (Education Science)(02),93-97.

[15] Wang Qianru, Zhang Rong.(2020). Analysis of stratification of network social circle from the perspective of sociology. Baoding college journal (06), 14 and 20.

[16] Chen $\mathrm{Yu}$ and $\mathrm{Chu}$ Hongsu.(2021). Social stratification of college students: External representation and development rules. Comparative Studies on Cultural Innovation (06),32-34. 